\title{
IMPROVEMENT OF ROAD TUNNEL VENTILATION THROUGH PREDICTIVE CONTROL
}

Design of an effective ventilation system for the road tunnel is usually a challenging task whose solution is less or more tending to optimum. Predictive control seems to be a promising approach that can help to improve properties of existing ventilation systems applied in road tunnels. Advantages of predictive control result mainly from its ability to solve both SISO and MIMO tasks, to have regard for dynamics of process changes in a broad extent, to compensate effect of measurable and non-measurable failures and to formulate the task as an "optimization control task" considering limiting conditions of control actions, changes of control actions and output variables. Data characterising the existing ventilation system can be used to analyse and identify the system and create its stochastic model. Thereafter the predictive control of ventilation can be designed enabling to predict concentrations of pollutants and optimize system operation.

\section{Introduction}

Predictive control is basically based on discrete or sampled models of processes. Therefore relevant relations and derivations are presented mainly in discrete area. The term "predictive control" denotes a class of control methods having a set of common properties: a mathematical model of the control system that is used for prediction of the future controlled output, known future trajectory of the required quantity, calculation of sequence of future control actions involving minimization of a proper cost function (usually quadratic) together with future trajectories of control increments and control deviation. Only the first proposed control action is performed and the whole minimization procedure is repeated in the next sampling period again. Usability of predictive control algorithms is quite wide and quality of control is usually higher than in the case of PID-controllers. They are applicable to unstable, multidimensional processes or processes with transport delay and compensate effects of measurable and non-measurable failures [1]. The road tunnel is also such a system since a proper algorithm must be used to keep concentrations of harmful pollutants under the certain level. To make a design of the predictive controller possible the existing tunnel system must be identified first using methods for system identification. Since each tunnel is unique, the design must be realised for the particular road tunnel - in this case for the Prague's urban tunnel Mrázovka. Data measured in the control centre of the tunnel are used to create basic types of stochastic parametric models and to realize them in the programme environment Matlab. Effects of ventilator operation on concentration of pollutants in the tunnel tube are also subject to identification.

\section{System identification}

For the purpose of identification it is interesting to describe the sought process using input-output relations [3]. The general procedure for estimation of the process model consists of several steps: determination of the model structure, estimation of parameters and verification of the model. Several stochastic models were considered as discussed below. In all of them existence of the stochastic component $\xi(\mathrm{t})$ was assumed. Most often we considered $\xi$ to be a white noise; however more complex cases are possible too. The stochastic models mentioned below are in discrete area all [3].

The model ARX (Auto-Regressive with eXogenous variable) assumes the error appearing as a white noise $\xi$ in the equation of the system:

$$
A y=B u+\xi .
$$

The model ARMAX (Auto-Regressive Moving Average with eXogenous Input) assumes the error appearing as the MA model, i.e.

$$
A y=B u+C \xi .
$$

In the case of the model OE (Output Error) we suppose that the stochastic component appears as a white noise additive to the output quantity (measurement noise).

$$
y=\frac{B}{A} u+\xi
$$

\footnotetext{
* Jozef Hrbcek, Ales Janota

Department of Control and Information Systems, Faculty of Electrical Engineering, University of Zilina, Slovakia,

E-mail: jozef.hrbcek@fel.uniza.sk
} 
where:

$w(k)=-a 1 w(k-1)-\ldots-a_{n a} w(k-n a)+b 1 \underline{u}(k-1)+\ldots+$ $+b_{n b} u(k-n b) ; y(k)=w(k)+\xi(k)$.

The structure Box-Jenkins gives a complete model with separately formed properties of failure from system dynamics. This model is suitable for those systems where failures are delayed in the process [3]:

$$
y=\frac{B}{A} u+\frac{C}{D} \xi .
$$

\subsection{Prediction of the output}

Estimation of the output $y_{P}(k)$ using a predictor is shown in Fig.1. Prediction of the output is usually a non-linear function $y_{p}(k)=f\left[y_{k-1}, u_{k-1}\right]$, where $y_{k-1}$ is a set of measured outputs, $u_{k-1}$ is a set of measured inputs.

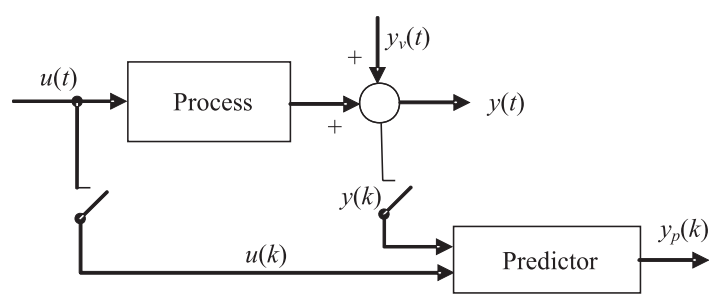

Fig. 1 Estimation of the output using a predictor

\section{Requirements for tunnel ventilation}

Tunnel ventilation is expected to fulfil the following requirements at least [5]:
- Concentration of emissions in the tunnel kept within the acceptable limits for the monitored harmful pollutants, in consideration of time spent by persons inside the tunnel;

- Good visibility for through passage of vehicles under polluted air inside the tunnel;

- Reduction of effects of smoke and heat on persons in the case of vehicle fire;

- Regulation of dispersion of pollutants in the air caused by petrol fumes from vehicles into the surround environment of the tunnel.

\subsection{Ventilation system of the Mrázovka road tunnel}

The ventilation system represents one function unit designed as longitudinal ventilation with a central efferent shaft and protection system avoiding spread of harmful pollutants into the tunnel surround area. Ventilation is longitudinal facing in direction of traffic with air suction at the south opening of the eastern tube (ETT) and at the branch B, with air being transferred at the north opening to the western tunnel tube (WTT) [7].

The task is to design a control system of ventilation based on traffic parameters, i.e. to find relationship between traffic intensity, speed of traffic and concentration of pollutants inside the tunnel. To do that the eastern tunnel tube (ETT) was chosen as a model example due to principle of mixing polluted air from ETT to WTT (measured concentrations of pollutants in the WTT are also influenced by traffic intensity in the ETT). To get the required description, the following data were taken from the tunnel: traffic intensity of trucks and cars, their speed, concentrations of $\mathrm{CO}$, NOx and visibility from the ETT. These concentrations are measured by sensors installed inside the tunnel (at five different places of the ETT). Traffic parameters are measured at three places, air flow at three places and concentrations of NOx in the north portal. Primary purpose of these measurements is to use data for ventilation control

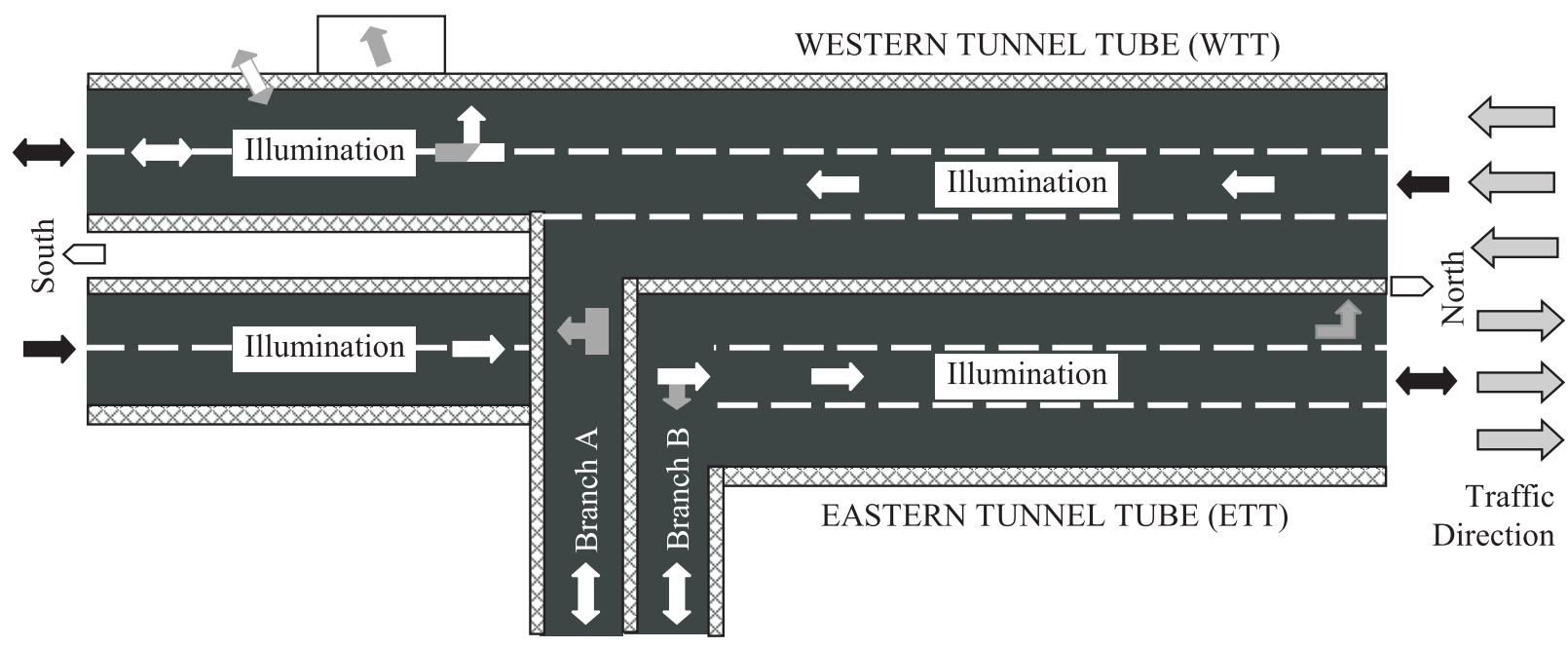

Fig. 2 Configuration of the road tunnel Mrázovka 
(switching ventilators) based on sensed level of emissions in the tunnel.

\section{Model Predictive Control (MPC)}

Model Predictive Control (MPC) comes from the late seventies when it became significantly developed [1] and several methods were defined. In this work we have applied the Dynamic Matrix Control (DMC) method which is one of the most spread approaches and creates the base of many commercially available MPC products. It is based on the model obtained from the transition or impulse characteristics:

$$
y(k)=\sum_{i=1}^{N} h_{i} u(k-i),
$$

Where hi are FIR (Finite Impulse Response) coefficients of the model of the controlled system. Predicted values may be expressed:

$$
\begin{aligned}
& \hat{y}(n+k \mid n)=\sum h_{i} \Delta u(n+k-i)+\hat{d}(n+k \mid n)= \\
& =\sum h_{i} \Delta u(n+k-i)+\sum h_{i} \Delta u(n+k-i)+ \\
& +\hat{d}(n+k \mid n)
\end{aligned} .
$$

We assume that the additive failure is constant during the prediction horizon:

$$
\hat{d}(n+k \mid n)=\hat{d}(n \mid n)=y_{m}(n)-\hat{y}(n \mid n) .
$$

Response can be decomposed to the component depending on future values of control and to the component determined by the system state in time $n$ :

$$
\hat{y}(n+k \mid n)=\sum h_{i} \Delta u(n+k-i)+f(n+k)
$$

Where $f(n+k)$ is that component which does not depend on future values of action quantity:

$$
f(n+k)=y_{n}(n)-\sum\left(h_{k+i}-h_{i}\right) \Delta u(n-i) .
$$

Predicted values within the prediction horizon $p$ (usually $p>>N$ ) can be arranged to the relation (4-5):

$$
\begin{aligned}
& \hat{y}(n+1 \mid n)=h_{1} \Delta u(n)+f(n+1) ; \\
& \hat{y}(n+2 \mid n)=h_{2} \Delta u(n)+h_{1} \Delta u(n+1)+f(n+2) ; \\
& \ldots \\
& \hat{y}(n+p \mid n)=\sum_{i=p-m+}^{p} h_{i} \Delta u(n+p-i)+f(n+p) .
\end{aligned}
$$

Where the prediction horizon is $k=1 \ldots p$, with respect to $m$ control actions. Regulation circuit is stable if the prediction horizon is long enough. The values may be arranged to the dynamic matrix $G$ :

$$
G=\left|\begin{array}{cccc}
h_{1} & 0 & \ldots & 0 \\
h_{2} & h_{1} & \ldots & 0 \\
\vdots & \vdots & & \vdots \\
h_{p} & h_{p-1} & \ldots & h_{p-m+1}
\end{array}\right|
$$

And expression used for prediction can be written in the matrix form:

$$
\hat{y}=G u+f,
$$

Where $\hat{y}$ is a vector of contributions of action quantity and $f$ are free responses. Test function is a quadratic criterion which can be expressed in the case of SISO (Single-Input Single-Output) system:

$$
\begin{aligned}
& J=\sum_{j=1}^{p}[\hat{y}(n+j \mid n)-w(n+j)]^{2}+ \\
& +\lambda \sum_{j=1}^{m}[\Delta u(k+j-1)]^{2}=e e^{T}+\lambda u u^{T}
\end{aligned}
$$

Where $e$ is a vector of future errors within the prediction horizon and $u$ is a vector of increments of the action quantity during the control horizon. The variant of DMC denoted as the Quadratic Dynamic Matrix Control (QDMC) is an optimization task solved numerically as a task of quadratic programming with limitations in the form of the system of linear inequalities:

$$
\begin{aligned}
& \sum_{i=1}^{p} C_{y i}^{j} \hat{y}(n+i \mid n)+C_{u i}^{j} u(n+i-1)+c^{j} \leq 0 \\
& j=1, \ldots, N_{c}
\end{aligned}
$$

Since future output values are connected to control values through the dynamic matrix $G$, the limiting condition can be expressed in the form:

$$
R u \leq c .
$$

\section{Simulations of predictive control in Matlab}

Fig. 3 shows a block diagram of the predictive closed-loop controller. Models of the tunnel and ventilator were obtained through identification of real equipment. Higher traffic intensity causes increase of pollutant concentrations in the tunnel. This intensity is expressed as a vector containing really measured data. The Matlab environment is used to simulate behaviour of the system according to Fig. 3. It is a closed-loop control (regulation) of the system with limitations imposed to control quantity and outputs. It uses MPC mode format and solves optimization problem with the use of quadratic programming. 


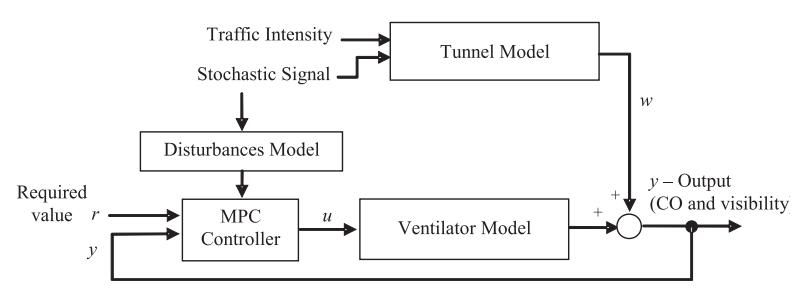

Fig. 3 Closed-loop control of ventilation using a predictive controler

\subsection{Components of the control circuit}

The "Ventilator Model" block is a model in the MPC step format that characterizes effect of ventilator on $\mathrm{CO}$ concentration and visibility (opacity). It is a system with 1 input $(u)$ and 2 outputs (dilution of $\mathrm{CO}$ concentration and opacity OP). One of the main advantages of predictive control is incorporation of limiting conditions directly to the control algorithm. In this case the control quantity $u$ is limited to the range $0-10 \mathrm{~V}$. The ventilator model has the form:

$$
\left[\begin{array}{l}
y_{1}(k) \\
y_{2}(k)
\end{array}\right]=\left[\begin{array}{l}
G_{1}(k) \\
G_{2}(k)
\end{array}\right] \cdot u(k)
$$

The "Tunnel Model" block is also a model in the MPC step format that is used to estimate a controller state. It enters the process as a vector of values calculated on the base of traffic intensity in a certain prediction horizon. Generally this model may also be expressed in a different form [8]. The "Disturbances Model" block has a MPC step format. Disturbing quantity in this simulation is a white noise. For each present and in future obtained setting of control actions $D u(k), D u(k+1), \ldots, D u(k+m+1)$, future behaviour of outputs of the process $y(k+1 \mid k), y(k+2 \mid k)$, ..., $y(k+p \mid k)$ can be predicted in the time horizon $\mathrm{p}$. Number of present and future control actions $m$ (while $m \leq p$ ) is calculated in such a way that the following quadratic criterion is minimized:

$$
\begin{aligned}
& \min _{\Delta u(k) \ldots \Delta u(k+m-1)} \sum_{l=1}^{p}\left\|\Gamma_{l}^{y}([y(k+l \mid k)-r(l+l)])\right\|^{2}+ \\
& +\sum_{l=l}^{m}\left\|\Gamma_{l}^{u}[\Delta u(k+l-1)]\right\|^{2}
\end{aligned}
$$

Where $\Gamma_{1}^{y}$ and $\Gamma_{l}^{u}$ are weighing matrices for evaluation of individual elements $y$ or $u$ in a certain time interval to future. $r(k+1)$ is a vector of future reference values (required value). Despite $m$ control actions was calculated (entire trajectory), only the first one $D u(k)$ is really used. In the next sampling period the whole procedure is repeated again. This principle is known as a strategy of moving horizon [3]. Predicted outputs of the process $\hat{y}(k+1 \mid k)$, ..., $(k+p \mid k)$ depend on actually measured quantity $y(k)$ and calculations will be performed with respect to non-measurable failures and measurable failures influencing outputs.
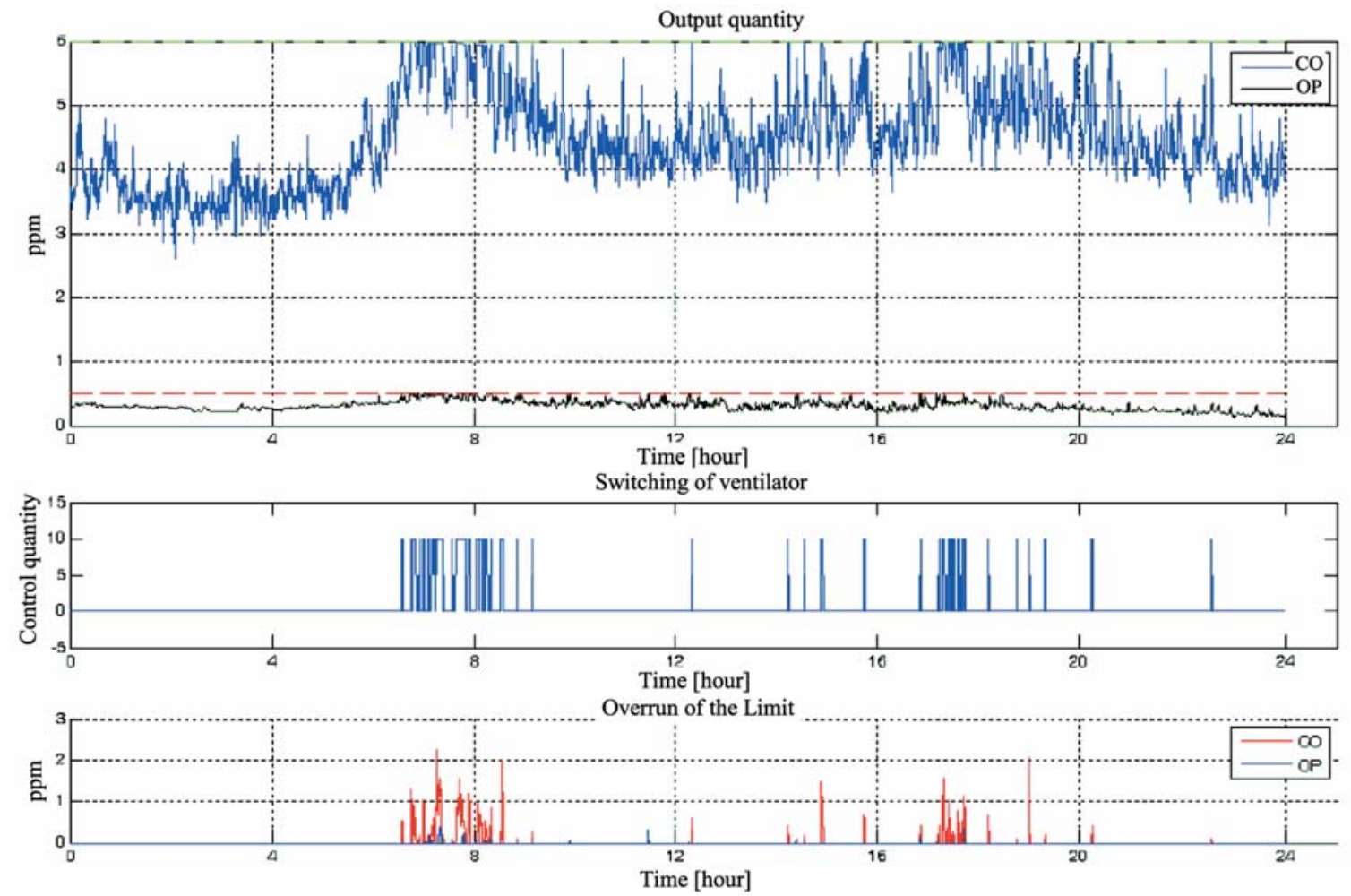

Fig. 4 Characteristics of MPC controller 


\subsection{Results of simulation}

The presented simulation results are obtained for the following concentration limits: $6 \mathrm{ppm}$ for $\mathrm{CO}$ concentrations and $0.5 \mathrm{ppm}$ for visibility concentrations. These values are below really defined maximum limits. According to the curve of the output quantity (Fig. 4) it is apparent that no emission value extended the defined limit. However, the value of under-set maximum limit may be extended since one ventilator need not be able to dilute $\mathrm{CO}$ concentration sufficiently. In the next simulations we used a possibility to set weighing matrices $(u w t)$ for tuning the controller. Correct setting of weighing matrices can avoid frequent switching of ventilators. In real operation the control quantity u must be adapted to the input of ventilator control unit.

\section{Conclusions}

The paper presents a methodology that was used for design of predictive control of road tunnel ventilation. Such a design needs identification of ventilator properties based on data obtained from the real ventilation system. Model of a two-dimensional system was created and simulated in Matlab environment using the method DMC. Presented results confirm higher effectiveness of predictive control approach where no monitored value extended the maximum set limits.

The paper was elaborated with support of the project SK123/CZ-108: ARICET.

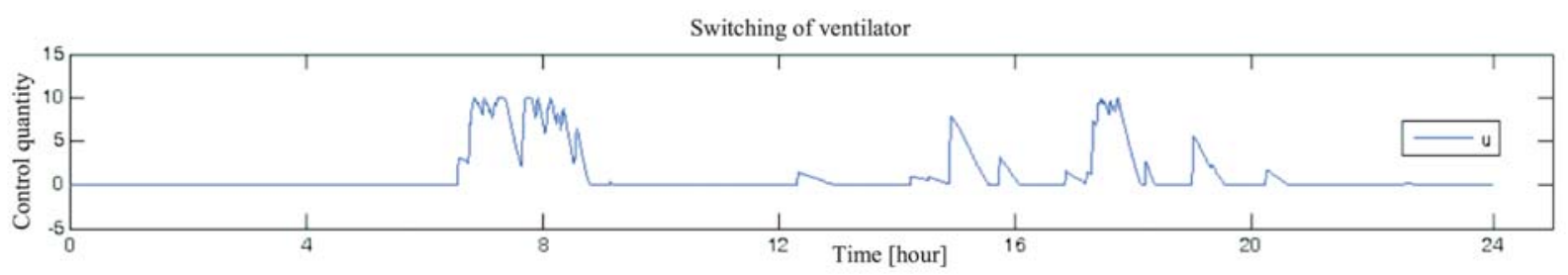

Fig. 5 The course of control quantity of the controller for uwt $=0.05$

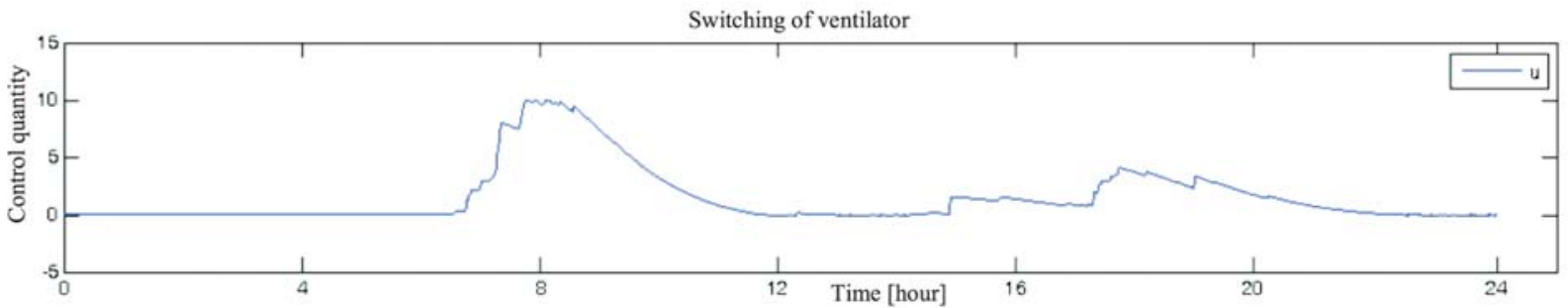

Fig. 6 The course of control quantity of the controller for uwt $=0.3$ and $y w t=[0.80 .8]$

\section{References}

[1] CAMACHO, E. F., BORDONS, C.: Model Predictive Control, $2^{\text {nd }}$ ed., Springer-Verlag London Limited, 2004, p. 405.

[2] MACIEJOVSKI, J. M.: Predictive Control with Constrains, 2002, p. 331

[3] JOHANSON, R.: System Modelling and Identification, Prentice-Hall, 1993, p. 512.

[4] HRBCEK, J.: Active Control of Rotor Vibration by Model Predictive Control - a Simulation Study, Report 153, Picaset Oy, Helsinki, 2007

[5] GODAN, J. et al.: Tunnels, Road Tunnels and Railway Tunnels (in Slovak), 2001, p. 202. 135882/p-UK/Z

[6] PRIBYL, P., JANOTA, A., SPALEK, J.: A nalysis and Risk Management in Traffic, Surface Traffic and Railroads (in Czech), BEN Praha, 2007, p. 515.

[7] PAVELKA, M., PRIBYL, P.: Simulation of Air Motion and pollutions inside the Road Tunnel - Mathematical Model (in Czech), OPTUN 228/06-EEG, 2006

[8] NOSKIEVIC, P.: System Modelling and Identification (in Czech), Ostrava, MONTANEX, 1999, p. 276, ISBN 80-7225-030-2. 